\title{
Detection of neuroendocrine tumors using promoter-specific secreted Gaussia luciferase
}

\author{
ALAN WEI-SHUN TSENG ${ }^{1,4}$, VICTORIA AKERSTROM ${ }^{1,2}$, CHIACHEN CHEN $^{1,2}$, \\ MARY B. BRESLIN ${ }^{1-3}$ and MICHAEL S. LAN ${ }^{1-3,5}$ \\ ${ }^{1}$ The Research Institute for Children, Children's Hospital, New Orleans, LA 70118; \\ ${ }^{2}$ Laboratory of Diana Helis Henry Medical Research Foundation, New Orleans, LA 70119; \\ Departments of ${ }^{3}$ Pediatrics, ${ }^{4}$ Biochemistry and Molecular Biology, and ${ }^{5}$ Genetics, \\ Louisiana State University Health Sciences Center, LA 70112, USA
}

Received August 20, 2015; Accepted October 6, 2015

DOI: $10.3892 /$ ijo.2015.3223

\begin{abstract}
Accurate detection of neuroendocrine (NE) tumors is critically important for better prognosis and treatment outcomes in patients. To demonstrate the efficacy of using an adenoviral vector for the detection of NE tumors, we have constructed a pair of adenoviral vectors which, in combination, can conditionally replicate and release Gaussia luciferase into the circulation after infecting the NE tumors. The expression of these two vectors is regulated upstream by an INSM1-promoter (insulinoma-associated-1) that is specifically active in NE tumors and developing NE tissues, but silenced in normal adult tissues. In order to retain the tumorspecificity of the INSM1 promoter, we have modified the promoter using the core insulator sequence from the chicken $\beta$-globin HS4 insulator and the neuronal restrictive silencing element (NRSE). This modified INSM1-promoter can retain NE tumor specificity in an adenoviral construct while driving a mutated adenovirus E1A gene ( $\triangle 24 E 1 A)$, the Metridia, or Gaussia luciferase gene. The in vitro cell line and mouse xenograft human tumor studies revealed the NE specificity of the INSM1-promoter in NE lung cancer, neuroblastoma, medulloblastoma, retinoblastoma, and insulinoma. When we combined the INSM1-promoter driven Gaussia luciferase with $\triangle 24 E 1 A$, the co-infected NE tumor secreted higher levels of Gaussia luciferase as compared to the INSM1p-Gaussia
\end{abstract}

Correspondence to: Dr Michael S. Lan, The Research Institute for Children, Children's Hospital, 200 Henry Clay Avenue, New Orleans, LA 70118, USA

E-mail:mlan@chnola-research.org

Abbreviations: NE, neuroendocrine; INSM1, insulinomaassociated-1; NRSE, neuronal restrictive silencing element; Luc2, luciferase2; Gau, Gaussia; Met, Metridia; MOI, multiplicity of infection; RFU, reflective fluorescence unit

Key words: adenovirus, detection, Gaussia, insulinoma-associated-1, neuroendocrine tumor virus alone. In a mouse subcutaneous xenograft tumor model, the combination viruses secreted detectable level of Gaussia luciferase after infecting an INSM1-positive NE lung tumor for $\geq 12$ days. Therefore, the INSM1-promoter specific conditional replicating adenovirus represents a sensitive diagnostic tool to aid clinicians in the detection of NE tumors.

\section{Introduction}

Neuroendocrine (NE) carcinomas are rare neoplasms that can develop into highly malignant and life-threatening tumors $(1,2)$. While they share a number of genetic and phenotypic traits, NE carcinomas comprise a very heterogeneous population of tumor types that can arise in various organs throughout the body. The most common of these cancers include neuroblastomas, retinoblastomas, medulloblastomas, pituitary carcinomas, small cell lung carcinomas, and carcinoid tumors, encompassing a broad spectrum of tumors that have so far required multiple detection and treatment methods (3-7). Despite their differences, many of these tumors express common tumor-specific markers that can identify them as NE cancers $(8,9)$. Consequently, early detection of these tumor markers can lead to better treatment response and outcomes. The INSMI gene encodes a NE tumor-specific marker that was discovered using an insulinoma subtractive hybridization screen $(10,11)$. The INSM1-promoter regulates the expression of INSM1, a transcription factor with a zinc-finger DNA binding domain that is highly specific for NE tumors (12). Through an Insml knockout mouse model, Insml transcription factor was found to be important in the formation of endocrine pancreas and sympatho-adrenal lineage during development $(13,14)$. Most interestingly, INSM1 expression was discovered to be restricted to the embryonic peripheral and central nervous system, specifically in the cells of neuroendocrine origin (15). The expression pattern was detected in the embryonic tissues of pituitary, pancreas, stomach, duodenum, thymus, adrenal glands, brain, and spinal cord, which were all found to be Insm1-positive at E15.5 in mice $(16,17)$. However, INSM1 is silenced in normal adult tissues, but reactivated in most of the human NE tumors, including neuroblastoma, medulloblastoma, pheochromocytoma, small cell lung carcinoma, 
insulinomas, pituitary tumors, carcinoid tumors, medullary thyroid carcinoma, and retinoblastoma (18). Therefore, INSM1 is a NE-specific tumor marker.

In order to assist with the detection of NE tumors despite their heterogeneous population, we have taken advantage of the INSM1-promoter's specificity in NE tumors to drive the expression of a downstream Gaussia luciferase gene. Secreted luciferases like Metridia or Gaussia luciferase have been shown to be highly luminescent, exhibiting 2-4-fold higher signal than Renilla or firefly luciferases $(19,20)$. We have constructed INSM1p-Met and INSM1p-Gau reporter vectors to measure the INSM1 promoter activity in NE tumors. In vitro cell lines and xenograft human tumor cultured cells revealed positive luciferase secreted from NE tumors. In addition, combining the INSM1p- $\triangle 24 E 1 A$ and INSM1p-Gau luciferase vectors increased the sensitivity of secreted Gaussia in vivo. The $\triangle 24 E 1 A$ gene, a mutant form of the adenovirus E1A gene with a 24-bp deletion, is inactive in retinoblastoma $(\mathrm{Rb})$ protein expressing cells and active in Rb-negative cancer cells (21). The cancer specificity from the modified INSM1 promoter and the $\triangle 24 E 1 A$ gene create a dual layer of safety against non-specific expression.

\section{Materials and methods}

Construction of adenoviral vectors. The Ad-INSM1p-Met construct was cloned using an original pGL3-INSM1p vector that contained the modified INSM1-promoter with HS4 insulator upstream and 2X NRSE downstream (22). The Metridia luciferase gene was excised from the pMetReporter vector (Clontech, Mountain View, CA, USA) and ligated downstream of the modified INSM1-promoter in pGL3. The pGL3 vector was cut to release the INSM1p-Met fragment, which was then ligated into the pShuttle plasmid (Agilent Technologies, Santa Clara, CA, USA) for adenoviral vector. The Ad-INSM1p-Gau and Ad-INSM1p- 24 EIA constructs were cloned using the modified INSM1-promoter on the pGL3-INSM1p vector, created by shortening the full insulator sequence into two copies of the core HS4 insulator. The Gaussia luciferase gene was obtained from the pMCSGaussia-Dura Luc vector (Thermo Fisher Scientific, Waltham, MA, USA) and ligated downstream of the INSM1-promoter to create pGL3-INSM1p-Gau. To clone the $\Delta 24 E 1 A$ gene, site directed mutagenesis was performed on an existing $E 1 A$ gene in the pJet plasmid (Thermo Fisher Scientific) to delete $24 \mathrm{bp}$ from the original sequence. This $\triangle 24 E 1 A$ gene was then cloned into the pGL3 vector to form pGL3-INSM1p- $\triangle 24 E 1 A$. Both the INSM1p-Gau and the INSM1p- $\Delta 24 E 1 A$ fragments were excised from their vectors and placed into the pShuttle plasmid. The Ad-SV40-Luc2 construct was generated by excising the SV40 promoter from the pSEAP2-Control vector (Clontech) and ligated upstream of the $L u c 2$ reporter gene in the pGL4.10 vector (Promega, Madison, WI, USA). The SV40-Luc2 fragment was cloned into the pShuttle vector. The pShuttle plasmid was linearized and electroporated into BJ5183-AD-1 cells (Agilent Technologies) to undergo recombination. After selection for the recombinants, linear adenoviral DNA was transfected into AD293 cells (Agilent Technologies) using FuGENE 6 reagent (Promega). The virus was amplified onto forty $150-\mathrm{mm}$ tissue culture dishes and purified by $\mathrm{CsCl}$ gradient. This purified virus was then titered using the Adeno-X-Rapid Titer kit (Clontech, Mountain View) and stored at $-80^{\circ} \mathrm{C}$. All sequences in the cloning process were verified through DNA sequencing.

In vivo luciferase imaging. $\mathrm{Nu} / \mathrm{Nu}$ mice (National Cancer Institute, Bethesda, MD, USA), aged 8-10 weeks, received intravenous tail vein injection of either the modified first generation Ad-INSM1p-Luc2, the second generation Ad-INSM1p-Luc2, or unmodified Ad-INSM1p-Luc2. The viruses were prepared in phosphate-buffered saline at a concentration of $10^{10} \mathrm{ifu} / \mathrm{ml}$ and $100 \mu \mathrm{l}$ of the viral solution was delivered slowly into the tail vein via a 27 -gauge needle. To perform the imaging analysis for luciferase activity, D-luciferin substrate (Biosynth, Itasca, IL, USA) was prepared at a concentration of $15 \mathrm{mg} / \mathrm{ml}$ and injected intraperitoneally into mice at a dose of $150 \mathrm{mg} / \mathrm{kg}$. Once injection was completed, the mice were anesthetized in an isofluorane chamber (2-4\% by inhalation) before being transferred to a Kodak In-Vivo Multispectral FX imager (Carestream Health, Rochester, NY, USA). Using the imager's software, luminescence was acquired with a 10-min exposure and an X-ray image of the mice in the same position was acquired with a 2-min exposure. Imaging was performed $48 \mathrm{~h}$ after virus injection and periodically for $\leq 28$ days. To generate the complete image, the luminescence acquisition was converted into a rainbow intensity scale and superimposed onto the X-ray acquisition using Image J software (National Institutes of Health, Bethesda MD, USA). For the NE tumor imaging, H1155 NE lung tumor cells $\left(1 \times 10^{7}\right)$ were pre-infected with the second generation modified Ad-INSM1p-Luc2 virus (50 MOI) for $24 \mathrm{~h}$ and injected subcutaneously into the right hind flank of nude mice $(n=3)$. After one week, the tumor growth was evidenced and imaged to show the modified INSM1 promoter specificity.

In vitro Metridia and Gaussia luciferase secretion assay. Cells were seeded in a 96-well plate at a density of 10,000 cells per well. After incubation at $37^{\circ} \mathrm{C}$ and $5 \% \mathrm{CO}_{2}$ for $1 \mathrm{~h}$, cells were infected with either no virus (negative control), Ad-INSM1p-Met (0-50 MOI), Ad-INSM1p-Gau (0-50 MOI), or Ad-SV40-Luc2 (5 MOI). Infected cells were then incubated at $37^{\circ} \mathrm{C}, 5 \% \mathrm{CO}_{2}$ for $24 \mathrm{~h}$. After incubation, each well was washed gently with $1 \mathrm{X}$ PBS and replaced with fresh media for another $24 \mathrm{~h}$. Fifty microliters of media per well were transferred to a 96-well white microplate. Luminescence was detected using the Pierce Gaussia Luciferase Glow Assay kit (Thermo Fisher Scientific), and read on a TopCount NXT Microplate Scintillation and Luminescence Counter. The adenoviral infection efficiency was determined by normalization (ratio) with intracellular luciferase (Ad-SV40-Luc2) using the Dual-Glo luciferase assay system (Promega) and read on a TopCount NXT Microplate Scintillation and Luminescence counter. To test the effects of Ad-INSM1p-Gau in combination with the Ad-INSM1p- $\Delta 24 E 1 A$ conditionally replicating adenovirus, cells were infected with a combination of $10 \mathrm{MOI}$ Ad-INSM1p-Gau and Ad-INSM1p- 24 E1A for a total of 20 MOI ( $2 \times 10^{5}$ ifu). Media was collected 2, 4, and 6 days (20 $\mu 1$ each day) after infection to determine secreted luciferase activity. Luminescence was detected using the Pierce Gaussia Luciferase Glow Assay kit (Thermo Fisher Scientific), 
then read on a TopCount NXT Microplate Scintillation and Luminescence Counter. The Student's t-test with a threshold of $\mathrm{p}<0.05$ was used to determine statistical significance. This process was repeated with the Ad-INSM1p-Gau infected cells to determine the Ad-INSM1p-Gau/Ad-SV40-Luc2 ratio.

Xenograft human tumor culture assay. Xenograft tumors were prepared by injecting human tumor cells $\left(1 \times 10^{7}\right)$, such as HeLa, U87, D283, UMC-11, SK-NBe(2), H1155, and H69 subcutaneously into the right hind flank of nude mice. Tumor tissues were harvested and frozen $\left(-80^{\circ} \mathrm{C}\right)$ in RPMI-1640 culture medium with $10 \%$ DMSO. The cultured tumor cells were prepared by rapidly thawing in a $37^{\circ} \mathrm{C}$ water bath and subsequent mincing into small sections $\sim 1 \mathrm{~mm}^{3}$ in size. The minced tissues were then centrifuged at $250 \mathrm{x} \mathrm{g}$ for $1 \mathrm{~min}$ and incubated in $2.5 \%$ trypsin for a total of $30 \mathrm{~min}$ at $37^{\circ} \mathrm{C}$ and $2 \mathrm{ml}$ growth media was added to neutralize the trypsin. The trypsinized tissues were then filtered through a $70-\mu \mathrm{m}$ sieve and centrifuged again at $250 \mathrm{x}$ g for $5 \mathrm{~min}$. The 96 -well clearbottom plates were coated with $75 \mu$ l per well of a 1:6 dilution of Matrigel in RPMI growth media and then incubated for $30 \mathrm{~min}$ at $37^{\circ} \mathrm{C}$. The tumor cells $(10,000$ cells) were re-suspended in RPMI media and added to each Matrigel coated well. Cells from each tumor were infected with Metridia-luciferase and Ad-SV40-Luc2 at $37^{\circ} \mathrm{C}$ for $24 \mathrm{~h}$. The ratio of Metridia/Luc 2 luciferase was calculated and averaged using RFU from $50 \mu \mathrm{l}$ media per well.

Detection of serum Gaussia luciferase in vivo. Eight-week-old $\mathrm{Nu} / \mathrm{Nu}$ mice (National Cancer Institute) were injected with H1155 NE lung tumor cells $\left(1 \times 10^{7}\right)$ subcutaneously into the right hind flank. Tumors were allowed to establish until tumor size grew to $\geq 0.1 \mathrm{~cm}^{3}$ in volume. The mice were injected intra-tumorally with $1 \times 10^{9}$ ifu of Ad-INSM1p-Luc2 virus, or a combination of $5 \times 10^{8}$ ifu of Ad-INSM1p-Gau and Ad-INSM1p- $\triangle 24 E 1 A$ (for a total of $1 \times 10^{9}$ ifu). To detect the Gaussia expression in the bloodstream, $100 \mu \mathrm{l}$ of blood was drawn at 3, 6, 9, and 12 days after virus injection. All animal experiments were performed in accordance with the approved protocol from the Institutional Animal Care and Use Committee, Louisiana State University Health Sciences Center New Orleans. The collected blood was allowed to clot for $30 \mathrm{~min}$ at room temperature and centrifuged at 2,000 $\mathrm{g}$ for $10 \mathrm{~min}$. Serum was collected from the supernatant and diluted with PBS at a 1:10 ratio. To detect Gaussia luciferase in the serum, $50 \mu \mathrm{l}$ of the diluted serum from each sample was added to a flat bottom 96-well plate for the Gaussia luciferase assay.

Statistical analysis. Values were corrected and expressed relative to a control group. All experiments were repeated three times. Results are presented as mean \pm SEM. Statistical analysis was performed using wither the Student's t-test when only two groups were in the experiment or by an one-way ANOVA comparison of multiple groups using the Tukey-Kramer test with differences at $\mathrm{p}$-value of $<0.05$ being considered significant.

\section{Results}

Cloning the INSM1-promoter driven adenoviral constructs. To generate an adenoviral vector that is useful for the diagnosis of
INSM1-positive NE tumors, we constructed the first generation modified INSM1-promoter by inserting a full HS4 insulator sequence upstream of the INSM1-promoter $(\sim 1.7 \mathrm{~kb})$ along with two NRSE enhancer sequences in tandem repeats downstream and Luc2 gene (Fig. 1A) (22). The modified INSM1-promoter drives a downstream Metridia luciferase gene, resulting in the construct Ad-HS4ins-INSM1p-2xNRSE-Metridia (Ad-INSM1p-Met) (Fig. 1B). A second generation of the modified INSM1-promoter was constructed to drive the expression of Gaussia luciferase and $\triangle 24 E 1 A$. This promoter was created using two copies of the HS4 core insulator in place of the full insulator sequence. The final constructs Ad-2xHS4CoreINSM1p-2xNRSE-Gaussia (Ad-INSM1p-Gau) and Ad-2xHS4Core-INSM1p-2xNRSE- 24 E1A (Ad-INSM1p$\triangle 24 E 1 A$ ), have a modified INSM1-promoter that is $\sim 700 \mathrm{bp}$ shorter than that of the promoter in Ad-INSM1p-Met (Fig. 1C and D). Ad-SV40-Luc2 vector was constructed as a control vector (Fig. 1E).

We tested whether an adenoviral vector driven by the modified INSM1-promoter would result in non-specific expression in vivo. Tail vein injection was performed using three viral vectors, the unmodified Ad-INSM1p-Luc2 (Fig. 1F), the first generation of the modified Ad-INSM1p-Luc2 (Fig. 1G), and the second generation modified Ad-INSMp-Luc2 (Fig. 1H) injected into non-tumor bearing $\mathrm{Nu} / \mathrm{Nu}$ mice separately. Therefore, we examined the INSM1 promoter specificity with or without HS4 insulator and NRSE enhancer sequence. After a period of 2-28 days, luciferase activity was determined via in vivo imaging system after intraperitoneal (i.p.) injection of luciferin substrate using a Kodak In-Vivo Multispectral FX imager. The intravenous injected adenovirus usually harbored in the liver ( $>90 \%)$. In the non-tumor bearing mice that were injected with the original unmodified Ad-INSM1p-Luc2, it was observed that non-specific luciferase expression occurred and was focused primarily in the liver area. In contrast, both the first and second generation modified Ad-INSM1p-Luc2 did not exhibit non-specific luciferase expression after luciferin administration. To further demonstrate that the modified Ad-INSM1p-Luc2 virus maintains NE tumor specificity, an Ad-INSM1p-Luc2 pre-infected H1155 NE tumor was established in nude mice and showed readily tumor imaging by luciferase (Fig. 1I). These results determined that the modified INSM1-promoter (both first and second generation) is essential in blocking the effects of adenoviral regulatory elements to retain tumor specificity in vivo.

Ad-INSM1p-Met displays INSM1 specificity in vitro. We constructed an adenoviral vector to express secreted Metridia luciferase specifically driven by the modified INSM1-promoter for the detection of NE tumors. Secreted Metridia was measured in vitro by co-infecting tumor cells with Ad-INSM1p-Met vector (Ad-HS4ins-INSM1p-2xNRSEMetridia) and Ad-SV40-Luc2. The addition of Ad-SV40-Luc2 virus was used to normalize the infection efficiency using the ratio between extracellular and intracellular luciferase activity. Both INSM1-negative and INSM1-positive tumor cell lines including lung carcinoma, neuroblastoma, medulloblastoma, pheochromocytoma, and insulinoma were infected with Ad-INSM1p-Met/Ad-SV40-Luc2 for 48 h (Fig. 2). The secreted luciferase activity in the media was readily detected in all of 


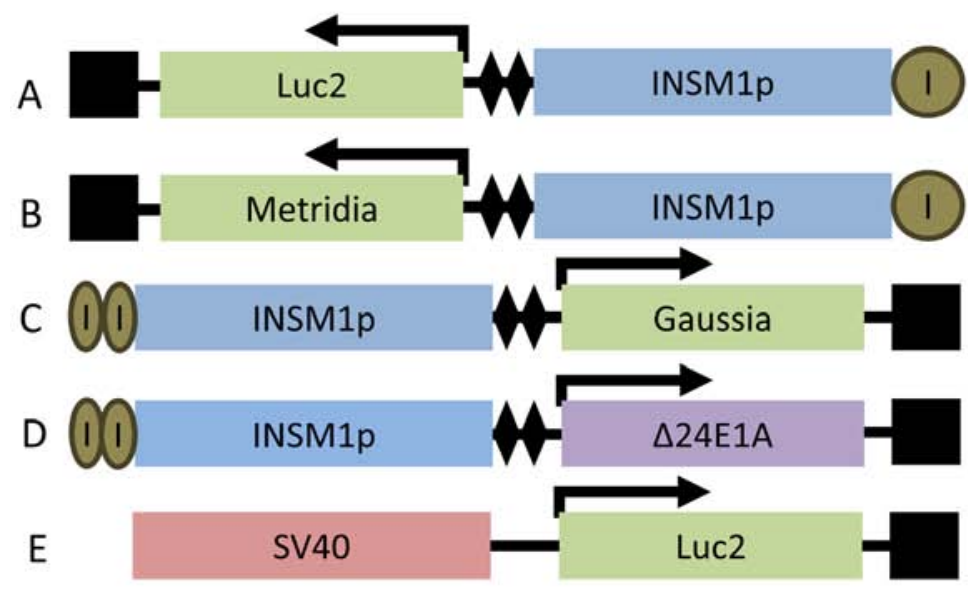

F

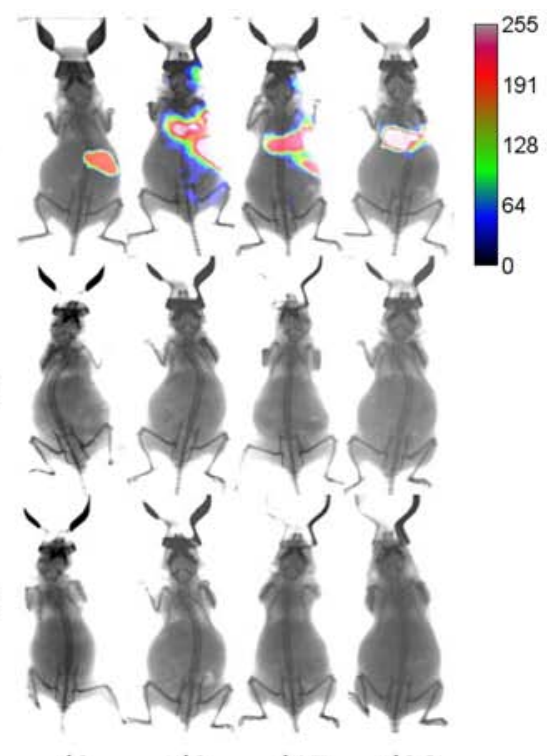

(1) (1) : $2 \times$ HS4 core insulator sequence

d2

d9

d17 $\quad$ d28

(1) : full HS4 insulator sequence

$\checkmark$ : NRSE $\square$ : poly-A tail

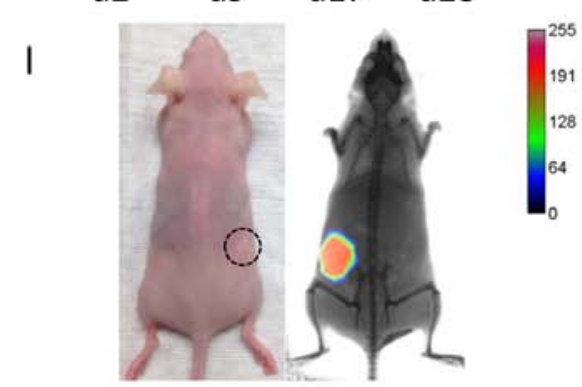

Figure 1. Adenoviral expression vectors. Adenoviral expression vectors were constructed using the SV40 and the modified INSM1 promoter. (A) The INSM1promoter driven firefly luciferase 2 vector was constructed using the first generation modified INSM1-promoter with a 1.2-kb HS4 insulator sequence and 2 copies of the NRSE regulator sequence (22). (B) The Metridia luciferase expression vector used the same first generation modified INSM1-promoter for the expression of Metridia luciferase. (C) The second generation INSM1-promoter was constructed using two tandem repeats of a 250-bp HS4Core insulator sequence in place of the full insulator sequence. This modified promoter was used to drive expression of Gaussia luciferase and (D) $\Delta 24 E 1 A$. (E) For positive control, the SV40 promoter was used to regulate the expression of firefly luciferase 2. For in vivo luciferase imaging, Nu/Nu mice aged 8-10 weeks received intravenous tail vein injection of either the unmodified or modified Ad-INSM1p-Luc2. (F) To test the specificity, unmodified INSM1-promoter driven Ad-INSM1p-Luc2 $\left(1 \times 10^{9}\right)$ was injected intravenously into non-tumor bearing $\mathrm{Nu} / \mathrm{Nu}$ mice and the luciferase signal was measured from days 2 to 28 post injection. $(\mathrm{G})$ The first generation and $(\mathrm{H})$ second generation modified INSM1-promoter driven Ad-INSM1p-Luc2 $\left(1 \times 10^{9}\right)$ do not show luciferase activity as INSM1 promoter retains its specificity. (I) For the NE tumor imaging, the modified Ad-INSM1p-Luc2 virus was pre-infected with H1155 NE lung tumor cells (50 MOI) and injected subcutaneously into the right hind flank of nude mice $(\mathrm{n}=3)$. After one week, the tumor growth was evidenced and imaged to show the modified INSM1 promoter specificity. Mouse tumor was faced down for luciferase imaging.

A

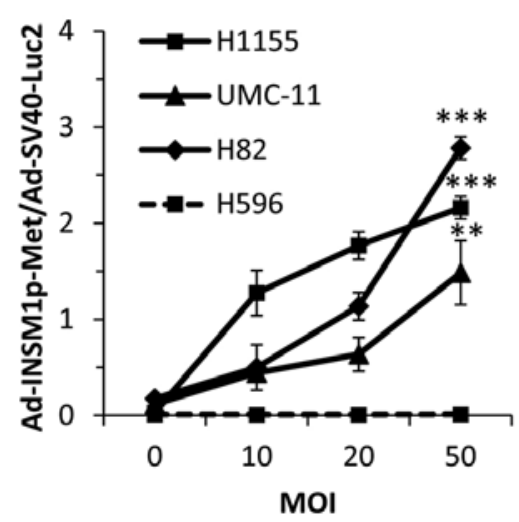

B

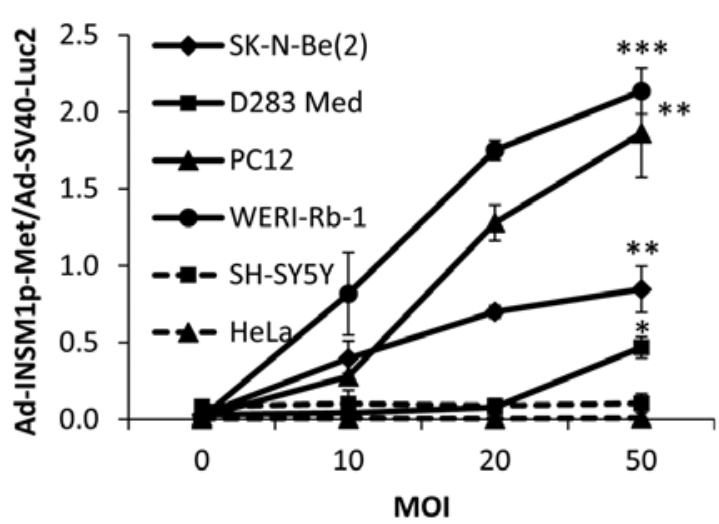

C

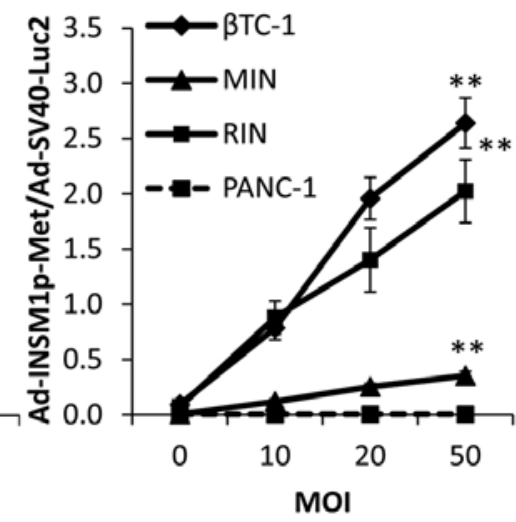

Figure 2. Ad-INSM1p-Met vector expressed Metridia luciferase specifically in INSM1-positive cell lines. An increasing Ad-INSM1p-Met concentration (0-50 MOI) and a constant Ad-SV40-Luc2 concentration (5 MOI) was used to infect INSM1-positive (solid lines) and -negative (dot lines) cell lines in culture. (A) NE lung cancer H1155, UMC-11, H82, and lung adenosquamous carcinoma H596 cells; (B) Neuroblastoma SK-N-Be(2) and SH-SY5Y, retinoblastoma WERI-Rb-1, pheochromocytoma PC-12, medulloblastoma D283 Med, and cervical adenocarcinoma HeLa cells; (C) Insulinoma $\beta$ TC-1, MIN, RIN, and pancreatic epithelioid carcinoma PANC-1 cells were used. Values are expressed as ratios between extracellular and intracellular luciferase activity. ${ }^{*} \mathrm{p}<0.05$, ${ }^{* * *} \mathrm{p}<0.01,{ }^{* * *} \mathrm{p}<0.001(\mathrm{n}=3)$. 


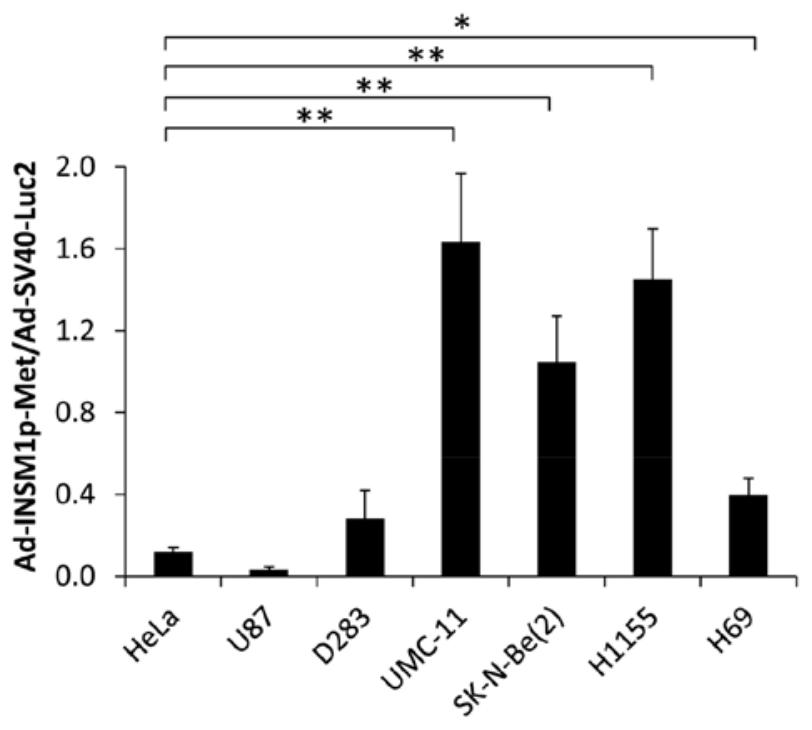

Figure 3. Metridia luciferase secretion in xenograft human tumor culture. Ad-INSM1p-Met vector specifically expressed Metridia luciferase in ex vivo NE tumor cells. Cells harvested from established xenograft human tumors were infected with Ad-INSM1p-Met (100 MOI) and AdSV40-Luc2 (100 MOI). Data points are displayed as ratios between extracellular and intracellular luciferase luminescence. ${ }^{*} \mathrm{p}<0.05,{ }^{* *} \mathrm{p}<0.01,{ }^{* * * *} \mathrm{p}<0.001(\mathrm{n}=4)$.

the INSM1-positive cell lines. In particular, the INSM1positive cell lines H82, $\beta$ TC-1, and WERI-Rb-1 exhibited the highest Ad-INSM1-Met/Ad-SV40-Luc2 luminescence ratios. At the highest $\mathrm{MOI}(50: 1)$, secreted luciferase activity reached $>2$-fold that of the intracellular luciferase activity (Fig. 2). In contrast, the INSM1-negative tumor cell lines showed no secreted Metridia luciferase relative to intracellular luciferase.

In order to assess the efficacy of INSM1-promoter driven Metridia luciferase adenoviral vector in xenograft human tumors, human tumor cultured cells derived from previously established xenograft tumor were collected and grown in culture. These ex vivo tumor cells were co-infected with Ad-INSM1p-Met and Ad-SV40-Luc2 to determine the ratio between extracellular and intracellular luciferase activity. After incubation for 3 days, it was determined that INSM1-positive cells [UMC-11, SK-N-Be(2), H1155, H69, except D283] infected by Ad-INSM1p-Met expressed extracellular Metridia luciferase that produced signals as high as 1.6 times the intracellular firefly luciferase (Fig. 3). In contrast, INSM1-negative cells (HeLa and U87) produced low levels of extracellular Metridia luciferase that did not exceed 0.11 times the activity of intracellular firefly luciferase.

INSM1 promoter-driven Gaussia luciferase retains specificity. In preparation for further in vivo assays, we switched from Metridia luciferase to a Gaussia luciferase expression vector due to the increased stability of Gaussia luciferase in vivo. As recent studies have shown, Gaussia luciferase signals were detectable after tail-vein injection in mice, due to its increased temperature stability compared to Metridia luciferase (19). To determine the specificity of our newly constructed Gaussia luciferase construct (Ad-INSM1p-Gau), we conducted an in vitro luciferase assay to evaluate whether our Ad-INSM1p-Gau vector (Ad-2xHS4Core-INSM1p2xNRSE-Gaussia) could specifically express Gaussia luciferase in INSM1-positive cell lines in a similar manner as our Ad-INSMp-Met vector. After co-infection with Ad-INSM1p-Gau/Ad-SV40-Luc2, we were able to see significant secreted luminescent activity in the media of all INSM1-positive cell lines as compared to INSM1-negative control cell lines (Fig. 4). In particular, the INSM1-positive cell lines $\beta$ TC-1, RIN, H82, and H1155 exhibited the highest Ad-INSM1-Gau/Ad-SV40-Luc2 ratios. Secreted Gaussia luciferase was not detected in any INSM1-negative cell lines, indicating INSM1 promoter retains specificity in vitro.

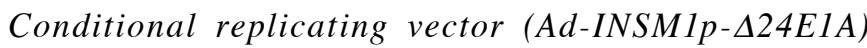
enhances Gaussia luciferase secretion and sensitivity over time in an in vivo mouse xenograft tumor model. After establishing the specificity of the Ad-INSM1p-Gau vector, we attempted to determine whether infecting cells with
A

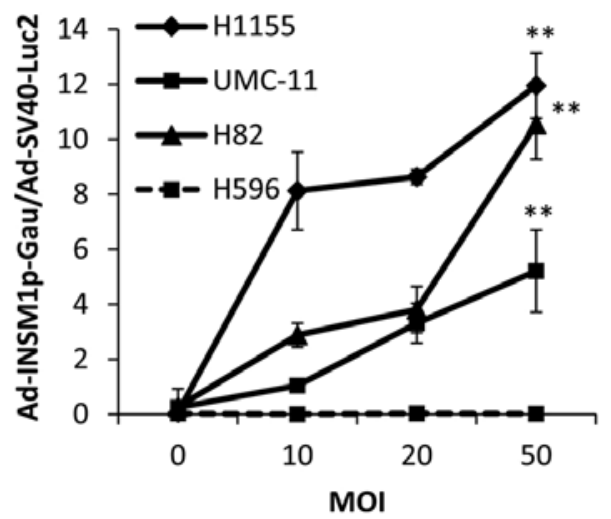

B

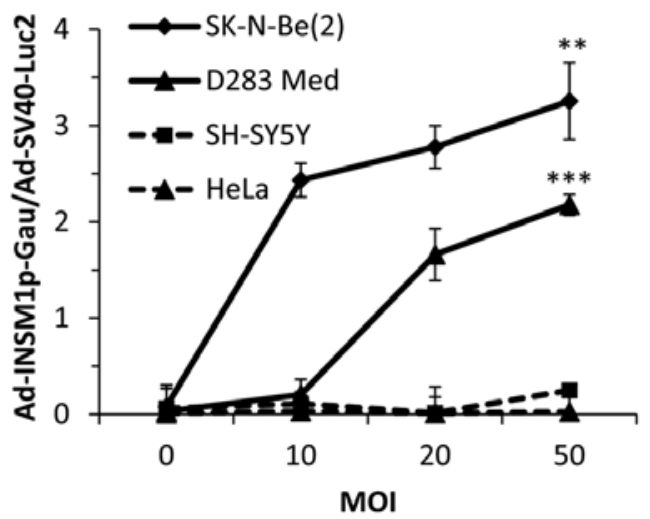

C

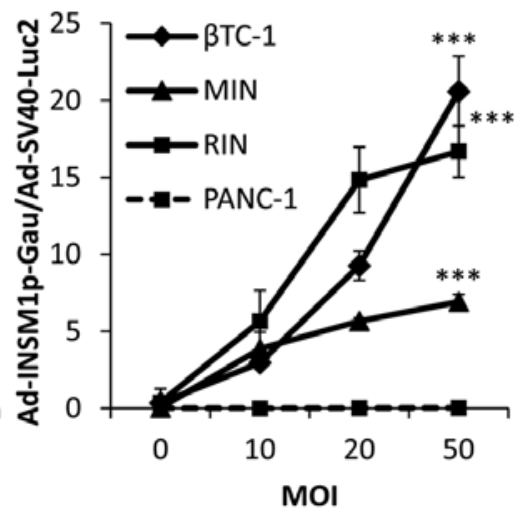

Figure 4. Ad-INSM1p-Gau vector expressed Gaussia luciferase specifically in INSM1-positive cell lines. An increasing Ad-INSM1p-Gau concentration (0-50 MOI) and a constant AdSV40-Luc2 concentration (5 MOI) was used to infect INSM1-positive (solid lines) and -negative (dot lines) cell lines in culture. (A) NE lung cancer H1155, UMC-11, H82, and lung adenosquamous carcinoma H596 cells; (B) Neuroblastoma SK-N-Be(2) and SH-SY5Y, medulloblastoma D283Med, and cervical adenocarcinoma HeLa cells; (C) Insulinoma $\beta$ TC-1, MIN, RIN, and pancreatic epithelioid carcinoma PANC-1 cells were used. Values are expressed as ratios between extracellular and intracellular luciferase activity. ${ }^{*} \mathrm{p}<0.05,{ }^{* *} \mathrm{p}<0.01,{ }^{* * * *} \mathrm{p}<0.001(\mathrm{n}=3)$. 


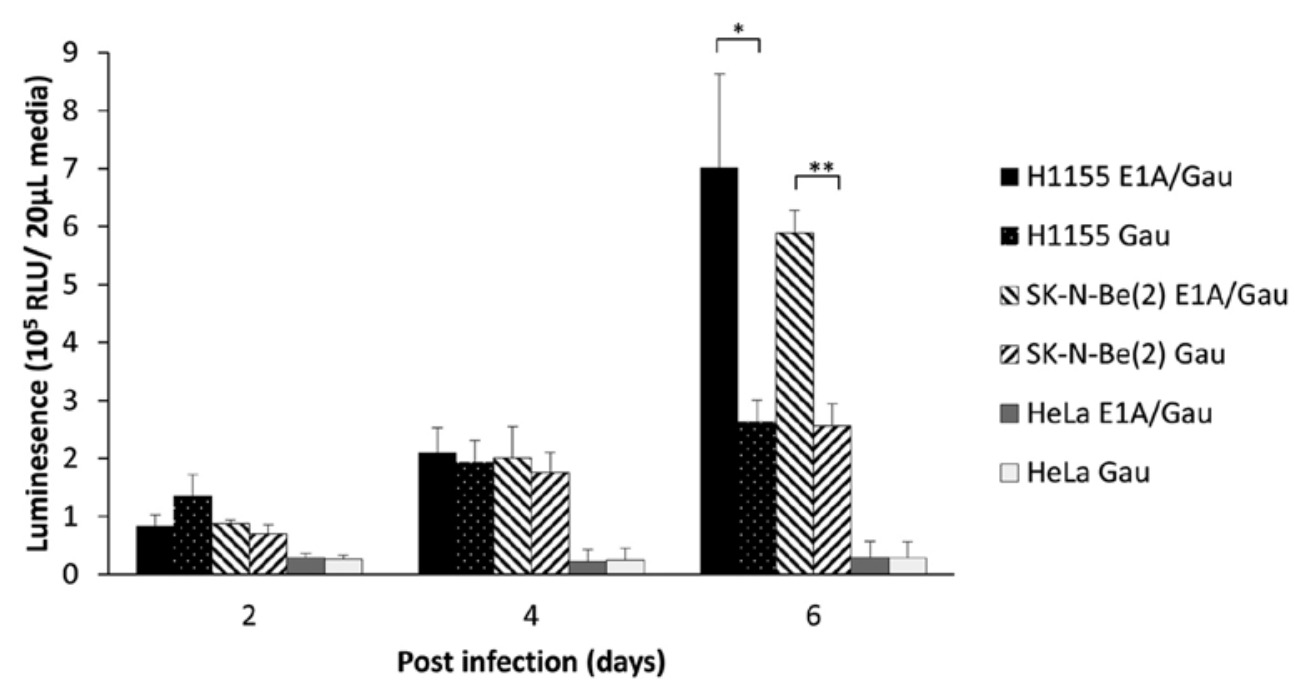

Figure 5. Oncolytic virus in combination with Ad-INSM1p-Gau increases sensitivity of Gaussia luciferase detection. The combination of Ad-INSM1p-Gau and Ad-INSM1p- $\triangle 24 E 1 A$ was able to increase the sensitivity of luciferase detection after 6 days post-infection. To test the effects of Ad-INSM1p-Gau in combination with the conditionally replicating adenovirus Ad-INSM1p- $\triangle 24 E 1 A$, cells were infected with combination of 10 MOI Ad-INSM1p-Gau and Ad-INSM1p- $\triangle 24 E 1 A$ (a total of $20 \mathrm{MOI}$ ). Media were collected 2, 4, and 6 days after infection and showed the highest luciferase activity at day 6. " $\mathrm{p}<0.05$, ${ }^{* *} \mathrm{p}<0.01(\mathrm{n}=3)$.

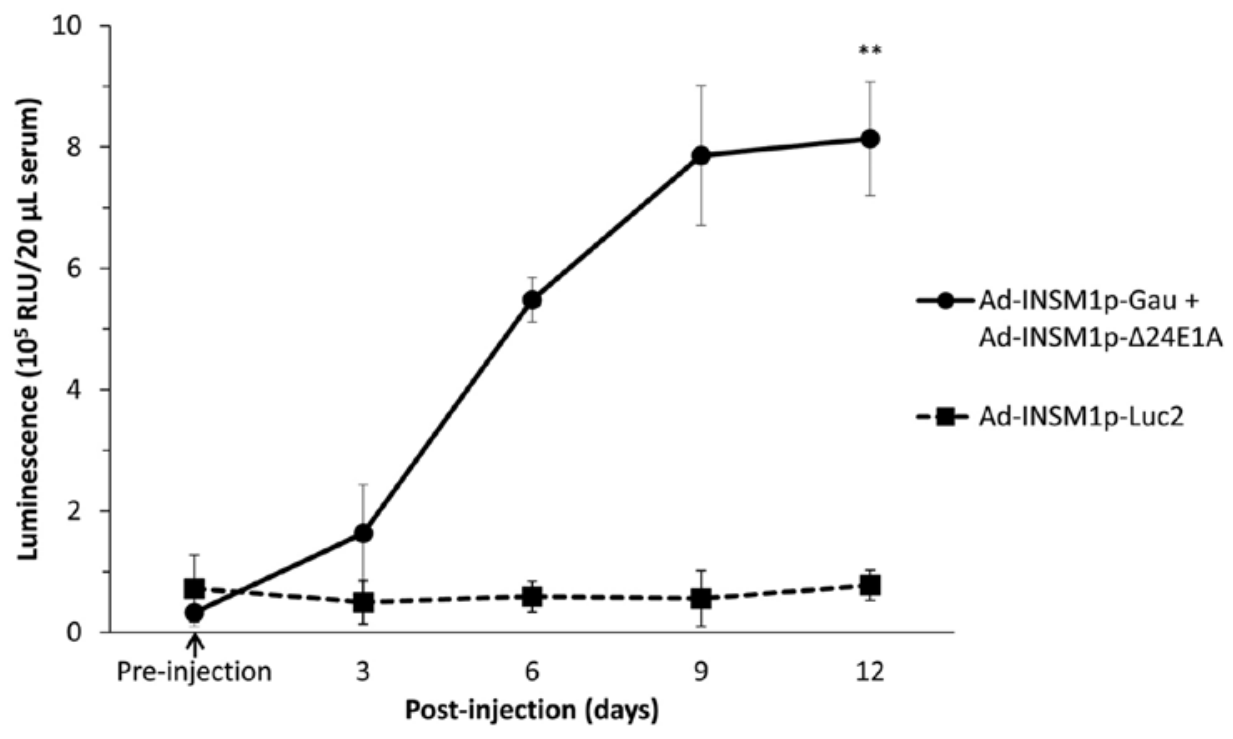

Figure 6. In vivo Gaussia secretion assay in a mouse xenograft tumor model. The combination of Ad-INSM1p-Gau and Ad-INSM1p- $424 E 1 A$ was able to express detectable Gaussia luciferase in circulation 12 days after infection in vivo. A mixture of Ad-INSM1p-Gau in combination with the Ad-INSM1p$\triangle 24 E 1 A$ conditionally replicating adenovirus ( $1 \times 10^{9}$ ifu total) was injected into established xenograft tumors (H1155 NE lung cancer cells). Serum was collected from each animal $(n=3) 3-12$ days post-infection. ${ }^{* *} \mathrm{p}<0.01(\mathrm{n}=3)$.

Ad-INSMp- $424 E 1 A$ in combination with Ad-INSM1p-Gau could increase the secreted luciferase signal. We hypothesized that if an INSM1-positive cell is infected concurrently with both viruses, $\triangle 24 E 1 \mathrm{~A}$ expression from Ad-INSM1p- $\triangle 24 E 1 \mathrm{~A}$ can be utilized by both viruses to facilitate replication. The replication of Ad-INSM1p-Gau should lead to viral amplification and increased Gaussia luciferase secretion over time. Therefore, we co-infected H1155 NE lung carcinoma cells or SK-N-Be(2) neuroblastoma cells with Ad-INSM1p-Gau alone $(20 \mathrm{MOI})$ or in combination with Ad-INSM1p- $\triangle 24 E 1 A$ at a concentration of $10 \mathrm{MOI}$ each (Fig. 5). Six days after infection, the combination viruses displayed a 2 -fold increase in secreted luminescent activity as compared to infection with Ad-INSM1p-Gau alone. This result suggested that our combination viruses could indeed amplify inside an INSM-positive cell in vitro.

We further analyzed whether the combination of Ad-INSM1p-Gau and Ad-INSM1p- $\triangle 24 E 1 A$ viruses could secrete detectable amount of Gaussia luciferase into the circulation from a tumor-bearing animal for an extended period of time. In this experiment, subcutaneous H1155 tumors $\left(\sim 0.1 \mathrm{~cm}^{3}\right)$ were first established on the right flank of $\mathrm{Nu} / \mathrm{Nu}$ mice. These tumor-bearing mice $(\mathrm{n}=3)$ were then injected intra-tumorally with either Ad-INSM1p-Luc2 or the 
Ad-INSM1p-Gau and Ad-INSM1p- $224 E 1 A$ virus combination at a total concentration of $1 \times 10^{9}$ ifu (Fig. 6). After infection with the virus combination for 6 days, detectable luciferase signal was observed in the serum $(p<0.01)$. The signal increased in intensity by day 9 and lasted up to 12 days, the humane endpoint for the tumor bearing animals. The Ad-INSM1p-Luc2 infected tumor released no Gaussia luciferase into the circulation and was used as the control.

\section{Discussion}

Although the original INSM1-promoter possesses NE-tumor specificity, it was discovered that the promoter loses its specificity when used in an adenoviral setting. In a recent study, Akerstrom et al demonstrated that an INSM1-promoter driven adenoviral reporter construct displayed non-specific expression after tail vein injection in an in vivo mouse model (22). It was hypothesized that this loss of specificity was due to the presence of overpowering viral enhancers that were otherwise not present in normal cells. To override these adenoviral regulatory elements, an insulator sequence derived from the HS4 chicken $\beta$-globin insulator was placed upstream of the INSM1-promoter to block effects from any viral enhancers. In addition, two copies of the neuron-restrictive silencer element (NRSE), a regulatory element with dual functions to silence the INSM1 promoter in non-neuronal cells while enhancing it in neuronal cells, were placed downstream of the promoter. Once these elements were added, the modified INSM1promoter was able to retain its high specificity in an adenoviral vector (22). To further improve upon this original design, the present study replaced the $1.2-\mathrm{kb}$ full insulator sequence with two copies of the HS4 core insulator (250 bp x 2) to create the second generation modified INSM1 promoter. Although the 1.2-kb full insulator sequence has been well characterized functionally, the 250-bp core insulator was observed to exhibit the same protective activity as the full sequence (23). The main benefit of switching from a full insulator sequence to the core sequence is that utilization of two copies of the 250-bp core would free $\sim 700 \mathrm{bp}$ of space for the assembly of larger transgenic sequences in the viral vector. Essentially, this more compact form of the modified INSM1-promoter displays the same NE tumor specificity with the additional advantage of allowing more flexible cloning strategies.

Retaining the specificity of the INSM1-promoter in an adenoviral vector has allowed us to construct a Gaussia luciferase reporter vector that can detect the presence of NE tumor in vivo. When paired with a conditionally replicating oncolytic virus, the virus combination allowed for continuous expression of Gaussia luciferase for the duration of the tumor's progression. These results could have a significant impact on monitoring tumor progression during the treatment of patients. Given that the viruses can selectively replicate in NE tumor cells, Gaussia luciferase expression should persist and intensify as the tumor increases in size. Conversely, if treatment of the tumor is successful, luciferase expression in the patient's blood should decrease as tumor size is reduced. Our study is a proof in principle that the Gaussia vector can be used in combination with a treatment protocol to monitor a patient's treatment outcome. An alternative use for this virus during the treatment of a NE tumor would be to discern whether a tumor is removed completely after surgical resection. By injecting the virus combination into the resection site during the surgical procedure, clinicians would be able to monitor the presence of INSM1-positive NE tumor cells based on a Gaussia luciferase readings from the patient's blood. Continuous monitoring of expression levels would allow for a better prognosis in these patients post-procedure by alerting clinicians to an incomplete resection.

Using the Ad-INSM1p-Gau vector in combination with the Ad-INSM1p- $\triangle 24 E 1 A$ was discovered to be more advantageous as compared to using Ad-INSM1p-Gau alone. In NE tumor cells infected by the virus combination, Gaussia luciferase expression was significantly higher than that of the Ad-INSM1p-Gau virus (20 MOI) alone after 6 days post-infection, even though the number of infectious units of Ad-INSM1p-Gau (10 MOI) was lower at the start for the combination. This indicates that the addition of $\triangle 24 E 1 A$ expression in cells infected by our Gaussia virus allowed for conditional replication of the reporter vector. This replication has the potential to significantly increase the copy number of the virus over several days, leading to an increase in sensitivity of Gaussia luciferase detection. Therefore, the most efficient method of increasing the sensitivity of infection seems to involve utilization of conditionally replicating viruses, as opposed to simply increasing the infectious units during administration of the virus.

Taken together, the Ad-INSM1p-Gau virus has the potential to be an easy-to-use and highly sensitive tool for the detection of NE tumors in the clinical setting. While a viral construct cannot be used as a diagnostic tool for the general population, it can be an alternative approach to track the tumor progression in patients with existing NE cancers. Additionally, it could also be used diagnostically in populations where a NE tumor is suspected. In these cases, the virus combination could act as both a diagnostic tool and as a way to monitor tumor progression.

\section{Acknowledgements}

This study was supported in part by the Research Institute for Children, Children's Hospital at New Orleans Louisiana.

\section{References}

1. Rindi G and Wiedenmann B: Neuroendocrine neoplasms of the gut and pancreas: New insights. Nat Rev Endocrinol 8: 54-64, 2012.

2. Jann H, Roll S, Couvelard A, Hentic O, Pavel M, MüllerNordhorn J, Koch M, Röcken C, Rindi G, Ruszniewski P, et al: Neuroendocrine tumors of midgut and hindgut origin: Tumornode-metastasis classification determines clinical outcome. Cancer 117: 3332-3341, 2011.

3. Brodeur GM, Pritchard J, Berthold F, Carlsen NL, Castel V, Castelberry RP, De Bernardi B, Evans AE, Favrot M, Hedborg F, et al: Revisions of the international criteria for neuroblastoma diagnosis, staging, and response to treatment. J Clin Oncol 11: 1466-1477, 1993.

4. Hayes FA, Green A, Hustu HO and Kumar M: Surgicopathologic staging of neuroblastoma: Prognostic significance of regional lymph node metastases. J Pediatr 102: 59-62, 1983.

5. Oyharcabal-Bourden V, Kalifa C, Gentet JC, Frappaz D, Edan C, Chastagner P, Sariban E, Pagnier A, Babin A, Pichon F, et al: Standard-risk medulloblastoma treated by adjuvant chemotherapy followed by reduced-dose craniospinal radiation therapy: A French Society of Pediatric Oncology Study. J Clin Oncol 23: 4726-4734, 2005. 
6. Packer RJ, Goldwein J, Nicholson HS, Vezina LG, Allen JC, Ris MD, Muraszko K, Rorke LB, Wara WM, Cohen BH, et al: Treatment of children with medulloblastomas with reduced-dose craniospinal radiation therapy and adjuvant chemotherapy: A Children's Cancer Group Study. J Clin Oncol 17: 2127-2136, 1999.

7. Richardson GE and Johnson BE: The biology of lung cancer. Semin Oncol 20: 105-127, 1993.

8. Mountain CF: Clinical biology of small cell carcinoma: Relationship to surgical therapy. Semin Oncol 5: 272-279, 1978.

9. Argiris A and Murren JR: Staging and clinical prognostic factors for small-cell lung cancer. Cancer J 7: 437-447, 2001.

10. Goto Y, De Silva MG, Toscani A, Prabhakar BS, Notkins AL and Lan MS: A novel human insulinoma-associated cDNA, IA-1, encodes a protein with 'zinc-finger' DNA-binding motifs. J Biol Chem 267: 15252-15257, 1992.

11. Lan MS, Russell EK, Lu J, Johnson BE and Notkins AL: IA-1, a new marker for neuroendocrine differentiation in human lung cancer cell lines. Cancer Res 53: 4169-4171, 1993.

12. Breslin MB, Zhu M, Notkins AL and Lan MS: Neuroendocrine differentiation factor, IA-1, is a transcriptional repressor and contains a specific DNA-binding domain: Identification of consensus IA-1 binding sequence. Nucleic Acids Res 30: 1038-1045, 2002.

13. Gierl MS, Karoulias N, Wende H, Strehle M and Birchmeier C: The zinc-finger factor Insm1 (IA-1) is essential for the development of pancreatic beta cells and intestinal endocrine cells. Genes Dev 20: 2465-2478, 2006.

14. Wildner H, Gierl MS, Strehle M, Pla P and Birchmeier C: Insm1 (IA-1) is a crucial component of the transcriptional network that controls differentiation of the sympatho-adrenal lineage. Development 135: 473-481, 2008.

15. Farkas LM, Haffner C, Giger T, Khaitovich P, Nowick K, Birchmeier C, Pääbo S and Huttner WB: Insulinoma-associated 1 has a panneurogenic role and promotes the generation and expansion of basal progenitors in the developing mouse neocortex. Neuron 60: 40-55, 2008.
16. Xie J, Cai T, Zhang H, Lan MS and Notkins AL: The zinc-finger transcription factor INSM1 is expressed during embryo development and interacts with the Cbl-associated protein. Genomics 80: 54-61, 2002.

17. Mellitzer G, Bonné S, Luco RF, Van De Casteele M, LenneSamuel N, Collombat P, Mansouri A, Lee J, Lan M, Pipeleers D, et al: IA1 is NGN3-dependent and essential for differentiation of the endocrine pancreas. EMBO J 25: 1344-1352, 2006.

18. Lan MS and Breslin MB: Structure, expression, and biological function of INSM1 transcription factor in neuroendocrine differentiation. FASEB J 23: 2024-2033, 2009.

19. El-Amouri SS, Cao P, Miao C and Pan D: Secreted luciferase for in vivo evaluation of systemic protein delivery in mice. Mol Biotechnol 53: 63-73, 2013.

20. Koutsoudakis G, Pérez-del-Pulgar S, González P, Crespo G, Navasa M and Forns X: A Gaussia luciferase cell-based system to assess the infection of cell culture- and serum-derived hepatitis C virus. PLoS One 7: e53254, 2012.

21. Whyte P, Buchkovich KJ, Horowitz JM, Friend SH, Raybuck M, Weinberg RA and Harlow E: Association between an oncogene and an anti-oncogene: The adenovirus E1A proteins bind to the retinoblastoma gene product. Nature 334: 124-129, 1988.

22. Akerstrom V, Chen C, Lan MS and Breslin MB: Modifications to the INSM1 promoter to preserve specificity and activity for use in adenoviral gene therapy of neuroendocrine carcinomas. Cancer Gene Ther 19: 828-838, 2012.

23. Aker M, Tubb J, Groth AC, Bukovsky AA, Bell AC, Felsenfeld G, Kiem HP, Stamatoyannopoulos G and Emery DW: Extended core sequences from the cHS4 insulator are necessary for protecting retroviral vectors from silencing position effects. Hum Gene Ther 18: 333-343, 2007. 